\title{
Framework for Flexible Multi-Metric Path Selection for Next Generation Network
}

\author{
Yevgeniy Yeryomin and Joc Hen Seitz
}

\begin{abstract}
This paper addresses the subject of multi-objective routing for an NGN environment. We propose a framework for a flexible multi- metric path selection which is designed as additional functional part for an NGN core. The framework allows the integration of user devices in NGN and a flexible definition and integration of a wide range of routing criteria. For the flexible definition of routing criteria, we propose a universal criteria raster. The proposed framework provides the users with the possibility of defining path selection rules between his devices and NGN core based on various sets of freely defined and parameterized criteria.
\end{abstract}

Index Terms-Next generation networks, flexible multi-metric routing, multi objective decision analysis, multi-constrained optimal path problem.

\section{INTRODUCTION}

This paper deals with flexible multi-metric path selection for NGN for the scenario where each user may have multiple end devices. In this introduction section we give a short overview of NGN, multiple-objective decision making and multi-metric routing.

The NGN is a technology trend of modern communication networks aimed to converge the communication systems in one unified system. NGN standardization is forced by ITU and 3GPP and described in general in [1] and 3GPP [2]. Some of the main characteristics of NGN are an IP-based core network, QoS support, compatibility with modern telecommunication networks and high level of mobility. A part of NGN is the fixed-mobile convergence (FMC) which targets at the consolidation of fixed and mobile networks. The main characteristics of FMC are the services delivery regardless of the fixed or mobile access technologies being used. FMC supports - by design defined as personal mobility - the use of several devices per user including multi-mode devices. FMC principles and general architecture are described in the ITU standardization recommendation [3].

As per design a user of an NGN/FMC network may have a number of communication devices for the same type of service. Each device represents a communication path towards its user. That fact results in a number of alternative paths between the NGN core and the user. That scenario raises the question how and based on which criteria a feasible and optimal path can be defined and determined. Path

Manuscript received August 12, 2014; revised November 30, 2014. This work is a part of a $\mathrm{PhD}$ project with the topic of flexible multi-metric path selection for the fourth generation networks.

The authors are with Communication Networks Group of Ilmenau University of Technology, Germany (e-mail: yyeryomin@yahoo.de, jochen.seitz@tu-ilmenau.de). selection is required prior to session establishment and during an established session for handover from one communication path to another. This problem can be considered as a special case of Multiple-Criteria Decision Analysis (MCDA) from the area of operational research. More specific problems relevant for the raised problem are referred to as Multi-Constrained Path Problem (MCP), Constraint-Based Routing (CBR) and Multi-Constrained Optimal Path Problem (MCOP). MCP and CBR are dealing with the calculation of feasible paths in a communication network, which satisfies certain constraints. The MCOP is a more general problem and includes MCP. MCOP is an optimization problem which deals with the determination of an optimal path from a number of feasible paths by taking into consideration a set of different criteria. A special case of CBR is the so called QoS-based routing problem where Quality of Service (QoS) characteristics are considered while determining the optimal path. A structured overview about MCP, MCOP and QoS-based routing algorithms is given in the papers [4], [5]. The proposed solutions are designed for global environments and for only for a fixed number of special metrics.

In this paper, we present a solution for flexible multi-metric path selection where metrics for the communication links and paths can be freely defined and parameterized and the path selection can be configured in a granular and flexible way. The proposed solution serves for the path selection between user and NGN core. The proposed system provides a universal criteria raster, in which a wide range of criteria may be integrated as long as these are quantitatively measurable and presentable. The delivery of criteria values is out of the scope of this paper. We assume that special agents/sensors exist on the communication nodes collecting the desired criteria values and making them available via documented open communication interfaces.

In the context of this paper the terms metrics and criteria have the same meaning.

The paper is structured a follows. Section II deals with the problem definition. Then, Section III presents an overview of the current state of related research in the area of multi-metric routing. In the next Section IV, the proposed system including its logical components is presented. In Section V, the validation of proposed system in a test environment is described.

\section{Problem Definition And Main ObJectives}

In an NGN environment, each user may have multiple devices. Also multi-mode devices can be used. In this case, a number of alternative communication paths presented by devices exist between the NGN core and a user as presented in 
Fig. 1. Since there are several alternative communication paths, the obvious problem is the determination of an optimal communication path to be used. For the calculation of the optimal communication path, a range of various criteria can be taken into account. As example, the criteria representing QoS or security parameters of communication paths or path priorities defined by user or service provider can be used. The criteria values for the link and path related criteria can be collected in special measurement points installed on the nodes of service provider, on nodes in the NGN core, on the user end devices. The values of criteria which are not related to the communication paths or links can be delivered by external application servers.

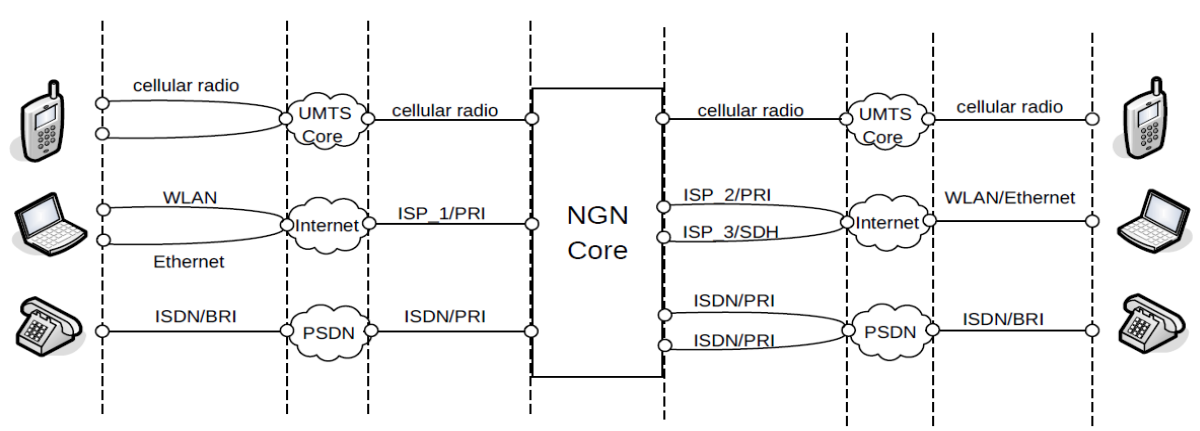

Fig. 1. A number of alternative communication paths in the NGN environment between users using multiple devices.

The path selection decisions both for incoming and outgoing calls, and for handover need to be made based on some kind of criteria. Depends on the direction the decision has to be made in the NGN core or on user device. The determination of optimal path between the NGN core and user with the multiple devices consists only of selection of the outgoing trunk in the NGN core or of selection of network interface on user device. The remaining routing decisions are performed by the service provider on the nodes in their networks.

For the selection of the optimal path, the paths and the links between the NGN core and user devices need to be determined. Since each user device represents a communication path to the user, the paths can be derived by the NGN core via the information of registered user devices. The link determination is more complex and requires the collaboration with the service provider.

The task of user or system administrator consists in defining the routing criteria, assigning them to known communication paths and links. The task of the system is the calculation of a restricted optimal path from the range of known paths by dropping not feasible paths, by calculation costs of each feasible path and by determining a path with the minimal cost.

To deliver a solution to the raised problem, the following points have to be addressed:

- Criteria definition;

- Determination of existing paths and links;

- Criteria values determination per path (end-to-end) and per link;

- Algorithms for determination of feasible optimal path based on multiple criteria.

In this paper, we propose a framework which enables both the user and the service provider to define the meaning of a feasible optimal communication path via a free and flexible parameterization of routing criteria. The system is designed as an additional platform for an NGN environment. The system provides a universal criteria raster for flexible criteria definition and allows the user to integrate devices in the system, to define the criteria and to configure the path selection between the NGN core and the user devices based on multiple criteria.

The proposed solution brings benefits both for end users to meet their service requirements and to optimize costs defined via criteria and for the service provider to optimize the network utilization.

\section{STATE OF THE ART}

In the current research, the routing techniques, where multiple criteria or metrics are used as constraints and for optimization, are referred to as QoS-based routing, security metric based routing, and constraint-based routing (CBR). All these routing techniques are considered as special cases of the MCP and MCOP problems from the area of operational research. MCP deals with the determination of communication paths, which satisfy certain service requirements. These paths are referred to as feasible paths. The aim of MCOP is the calculation of an optimal path from an amount of feasible paths. These problems for global environment are known as NP-hard.

The requirements for QoS-based routing are defined in RFC 2386 [6] as follows: dynamic determination of feasible paths, optimization of network utilization, network overload prevention. An overview of QoS-based routing algorithms is provided in [7]. These algorithms are designed to ensure some specific QoS parameters and to choose the optimal communication path in terms of QoS characteristics.

Security metric based routing is similar to QoS-based routing with the only difference that a security related metric is used for routing decisions. A routing metric based on network security aspects is investigated in [8]. A special kind of security based routing is the trust based routing for mobile ad hoc networks. For this kind of routing algorithms, the reputation of a node is calculated by observing the node's routing activities [9], [10].

CBR is described by IETF in [11] as a part of traffic engineering and defined as a class of routing protocols that make routing decisions to satisfy a range of constraints and requirements. CBR can also be used both for cost optimization and better network utilization. In [12], basic principles of CBR are explained and an overview of resent 
research is given. The aims of CBR are denoted in that paper as: cost reduction, network load balance and increase in security. The existing algorithms and solutions are designed for a global environment and for a limited set of criteria. As described in chapter 2 , we focus on a restricted environment and our aim is to design a flexible solution for nearly unlimited number of different criteria with high grade of flexibility in terms of criteria definition and multi-metric path selection configuration.

In [13], multi-metric routing for MANET has been investigated and a cross-layer Flexible Routing Decision framework has been proposed. This solution enables the configuration of multi-metric routing on per-application basis by taking into account MANET specific metrics.

In [14], a Distributed Multiple Criteria Routing extension to the distance vector algorithm is proposed. A Normalized Weighted Additive Utility Function (NWAUF) method has been developed. The NWAUF performs criteria value ranges normalization and multi-metric based metric calculation for routing entries.

A detailed overview of the vertical handoff decision strategies and algorithms for heterogeneous wireless networks is given in [15] and [16]. All techniques with exception of the Received Signal Strength (RSS) centric algorithms take into account multiple different criteria for the handoff decision. The strategies and algorithms are classified in groups described in following. Customer Based Strategies (CCS) where user defined policies used by handoff algorithm. In the Decision Function Based Strategies (DFBS) the weighted sum of all decision criteria for each communication path is calculated. Multiple Attribute Decision Making Strategies (MADMS) based on Multiple-criteria Decision Analysis (MCDA) methods like Simple Additive weighting (SAW), Technique for order preference by similarity to ideal solution (TOPSIS), Analytic Hierarchy Process (AHP) and Gray relational Analysis (GRA). In the Context Aware Strategies (CAS) application requirements are considered during handoff decision. The Fuzzy Logic and Neural Networks Based Strategies (FL/NN) deal with decision criteria containing inaccurate information.

The drawback of presented techniques is the lack of possibility to deal with criteria with non-linear value behavior. The non-linear criteria value transformation to a common scale provides a more exact optimization. The existing algorithms and methods don't satisfy the following requirements have been found:

- flexible definition and configuration of criteria

- flexible assignment of criteria to the paths

- feasible optimal path selection by consideration of both path and link constraints

- non-linear metric value transformation

- The next important subject of multi-metric routing is metrics or criteria used for routing decision. A comprehensive study on possible routing metrics has been conducted in [17]. The study worked out a detailed classification scheme for routing criteria and gave an overview about possible criteria. Four categories have been proposed: factor of influence, mathematical properties, design perspective and implementation characteristics. The category factor of influence divides criteria in two types depending on their relevance to the network: environmental and network-immanent.

Wang and Crowford mathematically investigated various QoS-metrics [18]. The criteria have been divided according to mathematical composition rules in three types: additive, concave and multiplicative.

A thorough analysis of QoS routing metrics for mobile ad-hoc networks (MANET) has been performed by Hanzo and Tafazolli [19]. The factors of influences on network performance have been studied and the metrics specific for physical, link, MAC, and network layers have been investigated. The ITU-T recommendation Y. 1540 [20] defines IP packet transfer performance parameters and service availability parameters.

Summarized, there is a great number of technology and ISO/OSI layer specific network characteristic which can be used as quantitative metrics for routing or path selection decision. Based on the metrics investigation, the universal criteria raster presented in Section IV.B was developed.

A next part of multi-criteria decision making is the need of criteria prioritization or weighting. The prioritization may be done completely manually or by support of the MCDM technique Analytic Hierarchy Process (AHP) [21]. AHP proposes a pairwise comparison of criteria in terms of their importance. The results of a comparison are stored in a matrix. By calculation of a ratio of the row sums, the priority or weight of each criterion can be determined.

\section{PRoposed SOLUTION}

At the beginning of this section, we give definitions of some terms used in the section below:

- user - NGN user owning a range of devices

- user devices - user terminals of different type, including so called multi-mode devices consisting of multiple different network interfaces (NI)

- multi-mode user devices - user device comprising several different network interfaces (NI)

- NGN core - NGN core infrastructure

- paths-communication paths between NGN core and user devices

- links-parts of communication paths created by the nodes on the path

- criteria-properties of different nature used for path selection

- path selection-determination of an optimal path based on a range of different criteria

- feasible link-a link satisfying given link constraints for all assigned criteria

- feasible path-a path consisting of only feasible links and satisfying given path constraints for all assigned criteria

- optimal path-a path with lowest cost

The system is designed as an additional component for an NGN environment to enhance it with flexible multi-metric path selection functionality. In this section, we describe the architecture of the proposed system and its components. The following main requirements have been defined for the proposed system: 
- high level of flexibility in terms of criteria definition and parameterization

- flexible criteria assignment to the communication paths

- selection of feasible optimal path based on multiple criteria

To meet the above mentioned requirements, the following system components have been defined:

- system logical components

- universal criteria raster

- configuration interface

- flexible multi-metric path selection algorithm

To store the information required for the path selection in a structured way, logical components like user, network trunk, criterion sensor connector, criterion and device have been developed. The universal criteria raster has been developed as a logical container for the criteria of different types and nature. The configuration interface is aimed to provide the users or system administrators with the ability of system parameterization. The mission of the flexible multi-metric algorithm is the calculation of paths costs based on multiple different criteria. These components are described in detail in the sections below.

\section{A. System Logical Components}

The proposed system contains user interface and core part.

The core consists of a range of logical components with relationships as presented in figure as presented in Fig. 2.

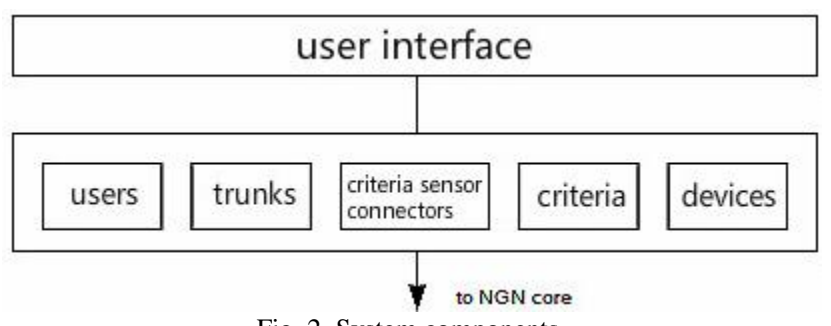

Fig. 2. System components.

To store all required information in a structured and modular form a range of logical components have been defined. Each logical component is designed to contain pieces of related information. Each component is a logical module, which can be filled with values for predefined parameters and associated with other components as presented in the relationship model in the Fig. 3.

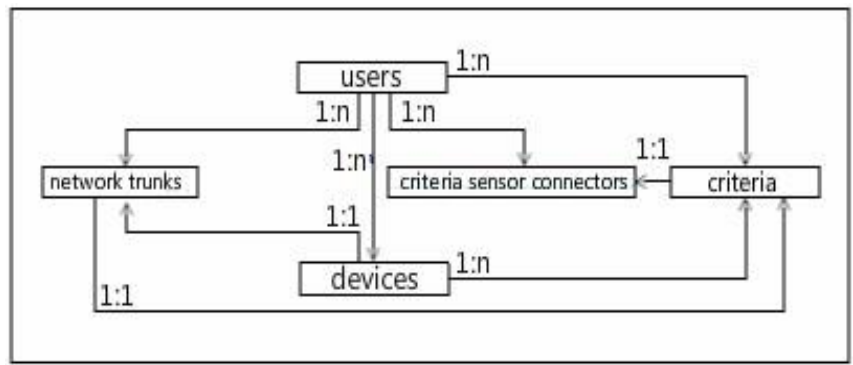

Fig. 3. Relationship model of system logical components.

As depicted in Fig. 3, user is central logical component. A user can own trunks, criteria driver, criteria and devices. Each device must have a trunk for connection to the NGN core. In our system, a device represents a communication path between the user and the core. Therefore, a device representing a communication path can own a range of criteria. Each criterion must own a criterion sensor connector. Below is a detailed explanation for each logical component:

- user - a central logical entity of the system representing users.

- trunk - physical or logical network connections from the underlying NGN environment to the access networks.The information about trunks has to be retrieved from the underlying NGN system.

- criteria sensor connector - interfaces to criteria value delivery agents. They parametrize the data delivered from the value delivery agents.

- criterion - one of an amount of routing criteria.

- device - user devices bound to a trunk. A range of various criteria could be assigned to a device.

The components are implemented in a relational database. The data contained in the components are used by the system for path selection. The data also can be used by the underlying NGN core for user and user devices management.

\section{B. Criteria Classification and Universal Criteria Raster}

The universal criteria raster is a logical entity, which gives user the ability to define, parameterize and prioritize a wide range of possible criteria. The criteria can be then applied to existing communication paths and links for the determination of the feasible and optimal path.

The essential requirements for a criterion are: a criterion needs to be quantitatively measurable and it needs to have an according criteria value delivery agent with an open communication interface. Each kind of criteria meeting these requirements can be integrated in the system.

TABLE I: UNIVERSAL CRITERIA RASTER

\begin{tabular}{|l|l|}
\hline \multicolumn{2}{|c|}{ Criteria sensor connector } \\
\hline \multicolumn{1}{|c|}{ Property } & \multicolumn{1}{c|}{ Value Type } \\
value type & $\begin{array}{l}\text { time } \\
\text { number } \\
\text { set } \\
\text { boolean }\end{array}$ \\
\hline original value type & numeric value range \\
\hline transformation function & $\begin{array}{l}\text { math function for value range } \\
\text { trasformation i.e. } \mathrm{f}(\mathrm{x})=2 \mathrm{x}\end{array}$ \\
\hline \multirow{3}{*}{ relevance } & $\begin{array}{l}\text { link related } \\
\text { path related } \\
\text { link and path related } \\
\text { global }\end{array}$ \\
\hline
\end{tabular}

\begin{tabular}{|l|l|}
\hline \multicolumn{2}{|c|}{ Criteria sensor connector } \\
\hline Property & \multicolumn{1}{|c|}{ Value Type } \\
\hline complexity & $\begin{array}{l}\text { atomar } \\
\text { complex }\end{array}$ \\
\hline link threshold & numeric value \\
\hline path threshold & numeric value \\
\hline weight (100 in total) & numeric value \\
\hline per path composition rule & $\begin{array}{l}\text { additive } \\
\text { concave } \\
\text { multiplicative }\end{array}$ \\
\hline
\end{tabular}

The universal criteria raster contains parameters to address constraint and optimization problems. For determination of feasible links and paths accordingly, path and link thresholds have been defined. To facilitate the calculation of the optimal path a normalized value range $K_{g}$, where $0<K_{g}<100$, and 
a criteria parameter for the transformation function have been introduced. The transformation function is a criteria specific mathematical function describing criteria value transformation from the original value range to the generalized value range $\mathrm{K}_{\mathrm{g}}$.

To ensure flexibility in terms of criteria configuration in the system, the universal criteria raster presented in the system has two logical components: the criteria sensor connector and the criterion. The criteria driver acts as an interface to a criteria value delivery agent. A criteria driver may belong to many criteria. Each criterion has to be assigned to a criteria driver. The universal criteria raster divided in criteria sensor connector and criteria is presented in Table I. The criteria driver parameters are described in details as follows:

- value type - specifies the type of the delivered value. The following types are defined: time, date, number, set, boolean. The value type set presents a set of alpha numeric values.

- original value range - specifies the criteria specific value range delivered from the criteria agent instance. Specification of the original value range is required only for numeric value types.

- transformation function - mathematical function specifying the transformation of the criteria specific value range to the system generalized criteria range. The system generalized criteria range is defined as $\mathrm{Kg}$, where $0<\mathrm{K}_{\mathrm{g}}<100$.

- relevance - defines the relevance of a criterion. A criterion may have link related, path related, link and path related or global nature. In case of a link and path related criterion, the criteria values for each link and path are to be provided by the criteria delivery agent.

The criterion component is the second part of universal criteria raster as presented in the Table I. The criterion component defines complexity, constraints and composition properties by means of following parameters:

- complexity - a criteria may be simple (atomic) or multidimensional (complex) when consisting of several sub-criteria.

- link threshold - acceptance boundary of a constraint for each link of a communication path. Path threshold acceptance boundary of a constraint for paths.

- composition weight - the weight is used to prioritize the criteria. The sum of weights of all criteria assigned to a path is 100. Prioritizing could be performed either manually or by use of a multi- objective decision technique Analytic Hierarchic Process (AHP).

- composition rule - defines the type of mathematical composition for criteria values across the path. Three composition rules are defined: additive, concave, and multiplicative.

The universal criteria raster described in this section allows definition of a wide range of various criteria which can be assigned to communication paths.

\section{Configuration Interface}

A configuration interface has been designed and developed as a WEB GUI. The configuration interface allows the user to parameterize the logical system components presented in the Section IV.A. To support the user during configuration, some component items like criteria sensor connector or criteria can be predefined by system administrator. The integration of a user and his devices and the configuration of criteria for path selection can be done in following steps:

1) Assignment of trunks provided by the underlying NGN core to the user.

2) Integration of user devices into the system. For each device a trunk to the appropriate network is assigned.

3) Assignment of predefined criteria sensor connector to the user or definition of new criteria driver.

4) Definition of new criteria, assignment of user criteria sensor connectors to the criteria.

5) Criteria prioritization manually or by means of AHP.

6) A range of criteria can be assigned to each user device (each device represents a communication path to the user).

Once a user, his devices and criteria are configured and parameterized, the constrained optimal path algorithm described in the next section can be used for the path selection.

\section{Flexible Multi-Metric Path Selection Algorithm}

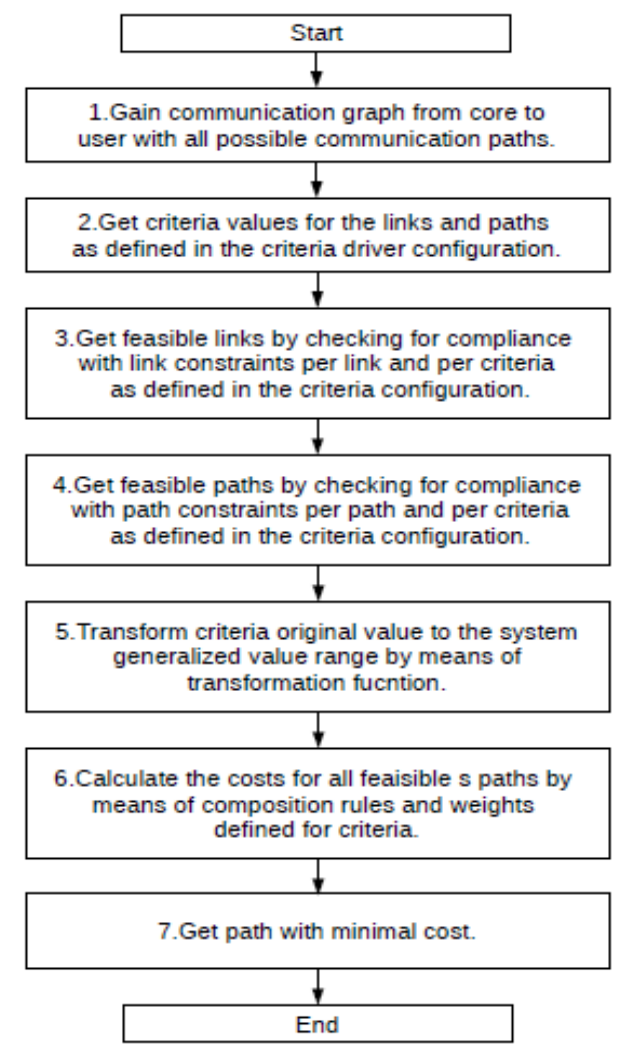

Fig. 4. Flexible multi-metric path selection algorithm.

For the calculation of the constrained optimal communication path, the constrained optimal path algorithm as presented in Fig. 4 has been developed and implemented in the proposed system. As the first step, the system determines the communication path and links to the user via configured user components like devices and trunks and the links information by collaboration with the service provider. As the second step, the system requests current path, link related and global values of assigned criteria from criteria sensors from network nodes and from external server. In the third and fourth steps, feasible links and paths are determined according to the configured thresholds. In the fifth step, the 
criteria values are normalized by transforming of criteria specific values to the generalized value range. In the sixth step, the costs for each communication path are calculated and in the last step the path with minimal costs is calculated.

\section{E. Formal Description of Flexible Multi-Metric Routing}

Given a set of communication paths, where $p \in P$. Each path $p$ contains a set of links $L_{p}$. Each $p$ is associated with a set of criteria $K_{p}$. The criteria from criteria set $K_{p}$ are associated with attributes from following sets:

- $P C_{k}$ - path constraint set

- $L C_{k}$ - link constraint set

- $P_{k}$ - priority set

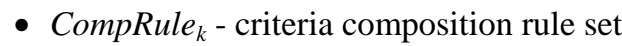

An optimal constrained path is calculated by following tree steps:

\section{1) Determination of feasible paths}

Check for link constraints for each link of each path:

$$
K_{i l} \leq L C_{i l}
$$

Check for path constraints for each path:

$$
\text { CompRule }_{i}\left(k_{i 1}, k_{i 2}, k_{i 3}, \ldots k_{i l}\right) \leq P C_{i}
$$

where: $i$ - criterion id; $l$ - link id.

\section{2) Value range transformation}

The criteria values for feasible paths are transformed to the system generalized value range in the following way:

$$
k_{i l}^{\prime}=f\left(k_{i l}\right)
$$

where:

$$
0 \leq f\left(k_{i l}\right) \leq 100
$$

\section{3) Path Cost Calculation}

Costs $W$ of each feasible path are calculated by use of slightly modified Weighted Sum Model (WSM) method [22] taken from Multi-Critera Decision Making (MCDM) research area. We added the composition rule CompRule as parameter in the WSM function for aggregation of link based criteria values across the path.

$$
W_{p}=\sum w_{i} \text { CompRule }_{i}\left(k_{i 1}^{\prime}, k_{i 2}^{\prime}, k_{i 3}^{\prime}, \ldots, k_{i e}^{\prime}\right)
$$

where $W$ - costs of a communication path; $n$ - the number of criteria; $w$ - criterion weight; $e$ - the number of links; $k$ criteria value, where $k_{l i}$ is the $i$-th criteria value of the first edge; CompRule - criterion composition rule (additive, concave, multiplicative).

The function composes the values of each criterion for all links across the path according to composition rule CompRule. In the next step the composed values of each criteria are summarized by means of criteria weight $w_{i}$. The result of the operations is the calculated path cost. Since we minimize the costs, the feasible path with minimum costs is an optimal path.

\section{SYSTEM VALIDATION}

To validate the proposed system, a demonstrator has been implemented in Java. The demonstrator contains a Web GUI and a database. The Web GUI is implemented in Java and ZK Studio and makes possible to configure the logical system components. The database is implemented in mySQL containing the tables for each logical system component presented in the Fig. 3. A call simulator has been implemented as a Java class. The call simulator facilitates the generation of a various number of calls towards a user. The Java class java.util.Random has been used for the criteria value generation in random manner.

\begin{tabular}{lccc}
\multicolumn{4}{c}{ TABLE II: THREE CRITERIA FOR PATH SELECTION ALGORITHM VALIDATION } \\
\hline \hline $\begin{array}{l}\text { Criteria driver } \\
\text { Value Type }\end{array}$ & $\begin{array}{c}\text { Criterion1 } \\
\text { (delay, mc) }\end{array}$ & $\begin{array}{c}\text { Criterion2 } \\
\text { (mon.costs,cent) }\end{array}$ & $\begin{array}{c}\text { Criterion3 } \\
\text { (power req., } \mathrm{W})\end{array}$ \\
$\begin{array}{l}\text { Original Value } \\
\text { Range }\end{array}$ & $0-400$ & number & number \\
$\begin{array}{l}\text { Transformatio } \\
\text { n Function }\end{array}$ & $\mathrm{x} / 4$ & $0-2000$ & $0-500$ \\
Relevance & link/path & link/path & link/path \\
\hline $\begin{array}{l}\text { Criterion } \\
\text { Complexity }\end{array}$ & atomar & atomar & atomar \\
Link Threshold & 40 & 40 & 40 \\
$\begin{array}{l}\text { Path Threshold } \\
\text { Composition }\end{array}$ & 100 & 100 & 100 \\
$\begin{array}{l}\text { Weight } \\
\text { Composition }\end{array}$ & 80 & 10 & 10 \\
Rule & additive & additive & additive \\
\hline \hline
\end{tabular}

Since the constrained part of the algorithm is pretty straightforward, the constraints have not been considered during the validation test. The optimization part of algorithms has been validated as described below.

TABLE III: CRITERIA WEIGHT CLASSES

\begin{tabular}{cccc}
\hline \hline Weight class & C1 & C2 & C3 \\
\hline 1 & 80 & 10 & 10 \\
2 & 33,33 & 33,33 & 33,33 \\
3 & 15 & 35 & 50 \\
\hline \hline
\end{tabular}

A test user with five devices had been configured in the demonstrator. Three additive criteria like delay, monetary cost and power requirements presented in Table II had been defined and assigned to user communication paths. We defined three weight classes for criteria as presented in the Table III.

TABLE IV: SUMS OF CRITERIA VALUES FOR RANDOM AND OPTIMAL PATH FOR SPECIFIC WEIGHT CLASSES FOR ONE THOUSAND CALLS

\begin{tabular}{ccccccc}
\hline \hline $\begin{array}{c}\text { Weight } \\
\text { class }\end{array}$ & C1 & C2 & C3 & C1 & C2 & C3 \\
\hline 1 & 48764 & 50176 & 47531 & 16332 & 45465 & 44064 \\
2 & 50630 & 48440 & 49832 & 30595 & 29679 & 30968 \\
3 & 48210 & 49705 & 50587 & 41091 & 30975 & 22331 \\
\hline \hline
\end{tabular}

TABLE V: SAVED SUMS OF CRITERIA VALUES FOR RANDOM AND OPTIMAL PATH FOR SPECIFIC WEIGHT CLASSES FOR ONE THOUSAND CALLS

\begin{tabular}{ccccccc}
\hline \multirow{2}{*}{$\begin{array}{c}\text { Weight } \\
\text { class }\end{array}$} & C1 & C2 & C3 & C1 & C2 & C3 \\
\hline 1 & 32432 & 4711 & 3467 & 66,51 & 9,39 & 7,29 \\
2 & 20035 & 18761 & 188646 & 39,57 & 38,73 & 37,86 \\
3 & 7119 & 18730 & 28256 & 14,77 & 37,68 & 55,86 \\
\hline \hline
\end{tabular}


One thousand calls for this scenario from NGN core to the user devices have been simulated. The criteria values were randomly generated in the system's internal value range from 1 to 100 for each communication link. We simulated three links for each communication path. For each call, the optimal communication path and a random communication path have been calculated. The sums of criteria for random and optimal communication path are shown in table IV. To outline the system benefits, the saved costs for the criteria sums of random and optimal path have been calculated and presented in the table $\mathrm{V}$. The table $\mathrm{V}$ demonstrates the saved costs for each weight class in system's internal units and in percents. The results show that the system has optimized the costs of each criterion according to criteria weights.

\section{OUTLOOK}

We consider as outstanding work the definition of criteria value delivery agents and criteria measurement techniques for particular criteria and their simulation and tests in the system. The normalization of metric value ranges is another point for further research. The normalization functions for special metrics need to be developed by consideration of criteria characteristics. Also the investigation of complex criteria is planned.

\section{CONCLUSION}

A In this paper, we analyzed flexible multi-metric path selection for the NGN scenario where each user may have multiple communication devices. The investigation of the state of the art showed that existing algorithms and solution are designed for a global environment and for a limited number of specific criteria. We developed and described in detail a framework for NGN which allows a multi-metric path selection in a flexible manner for a wide range of possible criteria. The system's logical components including a universal criteria raster were defined and described. The proposed system was validated in the implemented test environment and validation results are presented.

\section{REFERENCES}

[1] Next Generation Networks-Frameworks and Functional Architecture Models, ITU-T Recommendation Y.2001, 2004.

[2] Telecommunications and Internet converged Services and Protocols for Advanced Networking, NGN Release, ETSI TR 180001 v1.1.1, ETSI. 2006.

[3] Fixed-Mobile Convergence General Requirements, ITU-T Recommendation Q.1762/Y.2802, 2007.

[4] P. Nayak and G. R. Murthy, "Survey on constrained based path selection QoS routing algorithms: MCP and MCOP problems," Journal of Information Systems and Communication, 2013.

[5] S. Fahmy and O. Younis, "Constraint-based routing in the internet: Basic principles and recent research," IEEE Communications Surveys \& Tutorials, vol. 5, no. 1, 2003.
[6] E. Crawley, R. Nair, B. Rajagopalan, and H. Sandick, "RFC2386-A framework for Qos-based routing in the internet," IETF, 1998.

[7] K. Nahrstedt and S. Chen, "An overview of quality-of-service routing for the next generation high-speed networks: problems and solutions," IEEE Network: The Magazine of Global Internetworking, vol. 12, issue 6, pp. 64-79, 1998.

[8] I. A. Almerhag and M. E. Woodward, "Quality of service routing metrics based on selected aspects of network security," University of Bradford, 2005.

[9] N. K. Mohana, N. K. Srinath, and L. K. Amit, "Trust based routing algorithms for mobile ad-hoc network," International Journal of Emerging Technology and Advanced Engineering, vol. 2, issue 8, 2012.

[10] X. Li, M. R. Lyu, and J. Liu, "A trust model based routing protocol for secure Ad Hoc networks," in Proc. Aerospace Conference, 2004.

[11] D. Awduche, A. Chiu, A. Elwalid, I. Widjaja, and X. Xiao, "RFC3272 -overview and principles of internet traffic engineering," IETF, 2002.

[12] S. Fahmy and O. Younis, "Constraint-based routing in the internet: basic principles and recent research," IEEE Communications Surveys \& Tutorials, vol. 5, no. 1, pp. 2-13, 2003.

[13] K. Osathanunkul, "A cross-layer and multi-metric routing decision making framework for Manets," $\mathrm{PhD}$ dissertation, Faculty of Engineering and Physical Sciences, University of Manchester, 2013.

[14] B. Malakooti and I. Thomas, "Distributed composite multiple criteria routing using distance vector," in Proc. the 2006 IEEE International Conference on Networking, Sensing and Control, 2006, pp. 42-47.

[15] D. Cheelu, M. R. Babu, and P. V. Krishna, "A study of vertical handoff decision strategies in heterogeneous wireless networks," International Journal of Engineering and Technology, vol. 5, no. 3, pp. 2541-2554.

[16] A. Bhuvaneswari and E. G. D. P. Raj, "An overview of vertical handoff decision making algorithms," I. J. Computer Network and Information Security, vol. 9, pp. 55-62, 2012.

[17] M. Strasser, A. Weibel, R. Baumann, and S. Heimlicher, "A survey on routing metrics," Tik Report 262, ETH Zurich, Switzerland, 2007.

[18] Z. Wang and J. Crowcroft, "Quality-of-service routing for supporting multimedia applications," IEEE Journal on Selected Areas in Communications, vol. 14, pp. 1228-1234, 1996.

[19] L. Hanzo and R. Tafazolli, "A survey of QoS routing solutions for mobile ad hoc networks," University of Surrey, 2007.

[20] Internet Protocol Data Communication Service-IP Packet Transfer and Availability Performance Parameters, ITU-T Recommendation Y.1540, 2011

[21] T. L. Saaty, "Decision making with the analytic hierarchy process," Int. J. Services Sciences, vol. 1, no. 1, 2008.

[22] P. C. Fishburn, "Additive utilities with incomplete product set: applications to priorities and assignments," Operations Research Society of America, vol. 13, no. 3, pp. 537-542, May-June 1967.

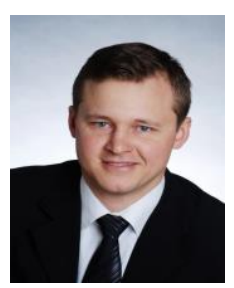

Y. Yeryomin completed his diploma engineer's in telecommunication engineering in year 2002. Since 2004 he is a member of Communication Networks Group of Ilmenau University of Technology in Germany. His research interests lie in the area of mobility for next generation networks (NGN) especially in the multi-criteria based path selection for NGN.

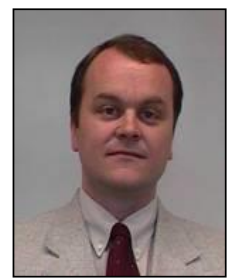

J. Seitz studied computer science at Universität Karlsruhe (TH) and got his Ph.D. degree from the same university in 1993 in the area of network management. After a post doc year at Lancaster University, Great Britain, and his postdoctoral lecture qualification (Habilitation), he became a professor of communication networks at Technische Universität Ilmenau in 2001. 